\title{
A genome-wide analysis of copy number variation in Murciano-Granadina goats
}

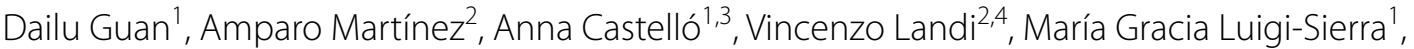 \\ Javier Fernández-Álvarez ${ }^{5}$, Betlem Cabrera ${ }^{1,3}$, Juan Vicente Delgado ${ }^{2}$, Xavier Such ${ }^{6}$, Jordi Jordana ${ }^{3}$ \\ and Marcel Amills ${ }^{1,3^{*}}$ (D)
}

\begin{abstract}
Background: In this work, our aim was to generate a map of the copy number variations (CNV) segregating in a population of Murciano-Granadina goats, the most important dairy breed in Spain, and to ascertain the main biological functions of the genes that map to copy number variable regions.

Results: Using a dataset that comprised 1036 Murciano-Granadina goats genotyped with the Goat SNP50 BeadChip, we were able to detect 4617 and 7750 autosomal CNV with the PennCNV and QuantiSNP software, respectively. By applying the EnsembleCNV algorithm, these CNV were assembled into 1461 CNV regions (CNVR), of which 486 (33.3\% of the total CNVR count) were consistently called by PennCNV and QuantiSNP and used in subsequent analyses. In this set of 486 CNVR, we identified 78 gain, 353 loss and 55 gain/loss events. The total length of all the CNVR (95.69 Mb) represented 3.9\% of the goat autosomal genome $(2466.19 \mathrm{Mb})$, whereas their size ranged from $2.0 \mathrm{~kb}$ to $11.1 \mathrm{Mb}$, with an average size of $196.89 \mathrm{~kb}$. Functional annotation of the genes that overlapped with the CNVR revealed an enrichment of pathways related with olfactory transduction (fold-enrichment $=2.33$, $q$-value $=1.61 \times 10^{-10}$ ), ABC transporters (fold-enrichment $=5.27, q$-value $=4.27 \times 10^{-04}$ ) and bile secretion (foldenrichment $=3.90$, $q$-value $=5.70 \times 10^{-03}$ ).

Conclusions: A previous study reported that the average number of CNVR per goat breed was 20 (978 CNVR/50 breeds), which is much smaller than the number we found here (486 CNVR). We attribute this difference to the fact that the previous study included multiple caprine breeds that were represented by small to moderate numbers of individuals. Given the low frequencies of CNV (in our study, the average frequency of CNV is $1.44 \%$ ), such a design would probably underestimate the levels of the diversity of CNV at the within-breed level. We also observed that functions related with sensory perception, metabolism and embryo development are overrepresented in the set of genes that overlapped with CNV, and that these loci often belong to large multigene families with tens, hundreds or thousands of paralogous members, a feature that could favor the occurrence of duplications or deletions by nonallelic homologous recombination.
\end{abstract}

*Correspondence: marcel.amills@uab.cat

${ }^{1}$ Centre for Research in Agricultural Genomics (CRAG), CSIC-IRTA-UAB-UB, Universitat Autònoma de Barcelona, 08193 Bellaterra, Spain

Full list of author information is available at the end of the article

\section{Background}

Copy number variations (CNV) encompass genomic deletions or duplications, with sizes ranging from 50 base pairs (bp) to several megabases (Mb), and which display polymorphisms (in terms of copy number) among individuals of a particular species [1-3]. In livestock, a broad array of phenotypes related with, among others, morphology [4, 5], pigmentation [6-9], sexual development 
[10] and susceptibility to disease [11] is caused by the segregation of CNV. Genome scans to detect structural variations in cattle have revealed that $\mathrm{CNV}$ regions (CNVR) are often enriched in genes that are involved in immunity [12-15], metabolism [12, 13], embryo development $[12,15]$ and sensory perception $[13,14]$. There is evidence that the $d_{N} / d_{S}$ ratios of genes that map to taurine $\mathrm{CNV}$ are generally higher than those of genes that do not overlap with $\mathrm{CNV}$, which indicates that $\mathrm{CNV}$ genes probably evolve under reduced selective constraint [13]. The analysis of gene networks has also shown that genes that co-localize with duplications tend to have fewer interactions with other genes than loci that do not overlap with $\mathrm{CNV}$, reinforcing the idea that genes mapping to duplicated regions have fewer essential housekeeping functions than non-CNV genes, and also have reduced pleiotropy [13].

Although structural chromosomal variations can have strong effects on gene expression and phenotypic variability, technical limitations and the moderate quality of genome assemblies have hampered CNV mapping in livestock [1]. Until recently, this has been particularly true for goats. In 2010, Fontanesi et al. [16] published the first caprine CNV map by identifying, with the Bovine $385 \mathrm{k}$ aCGH array, 127 CNVR including 86 and 41 copy loss and gain variants, respectively. Later on, resequencing the genome of individuals from several caprine breeds made it possible to identify $\mathrm{CNV}$ that overlap with 13 pigmentation genes and to detect an association between increased ASIP copy number and light pigmentation [17]. The first worldwide survey of copy number variation in goats was performed within the Goat ADAPTmap Project (http://www.goatadaptmap.org), and involved the genome-wide genotyping of 1023 goats from 50 breeds [18]. This study resulted in the identification of 978 CNVR among which several overlapped with genes that are functionally related with local adaptation such as coat color, muscle development, metabolic processes, and embryonic development [18]. Moreover, the patterns of the diversity of CNV differed according to geographic origin, which indicates that they have been influenced by population history [18]. In another study on 433 individuals from 13 East African goat breeds, Nandolo et al. [19] detected 325 CNVR. More recently, Henkel et al. [8] demonstrated the existence of complex patterns of structural variation in the regions containing the caprine ASIP and KIT genes, with potential causal effects on pigmentation. In spite of these efforts, the description of structural chromosomal variation in goats is still lagging behind that of other domestic species. Most of the CNV surveys in goats have analyzed large populations that represent a mixture of different breeds each with a limited number of individuals $[18,19]$, thus making it difficult to assess the magnitude of the CNV diversity at the within-breed level. Our goal was to fill this gap by analyzing a population of 1036 individuals from a single Spanish breed (MurcianoGranadina), and to investigate the functional roles of genes that map to CNVR and compare these results with data obtained in composite goat populations.

\section{Methods}

Genomic DNA extraction and high-throughput genotyping Blood samples from 1036 Murciano-Granadina female goats from 15 farms that are connected through the use of artificial insemination were collected in EDTA $\mathrm{K} 3$ coated vacuum tubes and stored at $-20{ }^{\circ} \mathrm{C}$ before processing. Genomic DNA was isolated by a modified salting-out procedure [20]. Four volumes of red cell lysis solution (Tris- $\mathrm{HCl} 10 \mathrm{mmol} / \mathrm{L}, \mathrm{pH}=6.5$; EDTA $2 \mathrm{mmol} / \mathrm{L}$; Tween $201 \%$ ) were added to $3 \mathrm{~mL}$ of whole blood, and this mixture was centrifuged at $2000 \times \mathrm{g}$. Pelleted cells were resuspended in $3 \mathrm{~mL}$ lysis buffer (Tris$\mathrm{HCl} 200 \mathrm{mmol} / \mathrm{L}, \mathrm{pH}=8$, EDTA $30 \mathrm{mmol} / \mathrm{L}$, SDS 1\%; $\mathrm{NaCl} 250 \mathrm{mmol} / \mathrm{L})$ plus $100 \mu \mathrm{L}$ proteinase $\mathrm{K}(20 \mathrm{mg} /$ $\mathrm{mL}$ ). The resulting mixture was incubated at $55{ }^{\circ} \mathrm{C}$ for 3 $\mathrm{h}$ followed by centrifugation at $2000 \times g$ in the presence of $1 \mathrm{~mL}$ of ammonium acetate $(10 \mathrm{~mol} / \mathrm{L})$. The supernatant $(\sim 4 \mathrm{~mL})$ was mixed with $3 \mathrm{~mL}$ of isopropanol $96 \%$, which was subsequently centrifuged at $2000 \times g$ for $3 \mathrm{~min}$. The supernatant was removed and the DNA pellet was washed with $3 \mathrm{~mL}$ of ethanol 70\%. After centrifuging at $2000 \times g$ for $1 \mathrm{~min}$, the DNA precipitate was dried at room temperature and resuspended in $1 \mathrm{~mL}$ of TE buffer $(10 \mathrm{mmol} / \mathrm{L}$ Tris, $\mathrm{pH}=8.0 ; 1 \mathrm{mmol} / \mathrm{L}$ EDTA, $\mathrm{pH}=8)$.

High-throughput genotyping of the 1036 MurcianoGranadina DNA samples was carried out with the Goat SNP50 BeadChip [21] according to the manufacturer's instructions (Illumina). Signal intensity ratios i.e. $\log R$ Ratio or LRR (the total probe intensity of a SNP referred to a canonical set of normal controls [22]), and B allele frequencies or BAF (relative quantity of one allele compared to the other one) [22], were exported for each single nucleotide polymorphism (SNP) with the GenomeStudio software 2.0.4 (Illumina, https://emea.illumina. com). Then, SNP coordinates were converted to the latest version of the goat reference genome (ARS1) [23]. After filtering out unmapped and non-autosomal SNPs and those with a call rate lower than $98 \%$, a set of 50,551 SNPs remained for $\mathrm{CNV}$ mapping.

Copy number variant calling with PennCNV and QuantiSNP Based on their excellent performance in comparative studies, we selected two software packages, PennCNV v1.0.5 [24] and QuantiSNP v2 [25], to call CNV in the Murciano-Granadina population [26, 27]. The PennCNV software [24] detects CNV by applying the default 
parameters of the Hidden-Markov model. Population frequencies of B alleles were compiled based on the BAF of each SNP in the population. We used the "-gcmodelfile" option to adjust "genomic waves" [28]. The number of goat chromosomes was set with the "-lastchr 29" instruction. The QuantiSNP analysis [25] assumes an objective Bayes hidden-Markov model to improve the accuracy of segmental aneuploidy identification and mapping. This CNV calling software was run under default parameters by modifying the "-chr 1:29" option. The CNV that were supported by less than three SNPs were removed from the filtered set used here.

\section{Definition and functional annotation of copy number variant regions}

We used the EnsembleCNV algorithm (beta version) [29] to assemble CNVR. All CNV called by PennCNV and/ or QuantiSNP were combined to generate a set of initial CNVR by using the heuristic algorithm (threshold of minimum overlap $=30 \%$ ) described in [29]. Subsequently, CNVR boundaries were refined by considering the local correlation structure of the LRR values of the SNPs mapping to CNVR [29]. Then, we reassigned the $\mathrm{CNV}$ calls that were initially obtained with PennCNV and QuantiSNP to each refined CNVR, so that the final set of CNVR comprised only those that were simultaneously detected by both callers. The resulting CNVR were matched to gene features that are annotated in the National Center for Biotechnology Information (NCBI, https://www.ncbi.nlm.nih.gov) by using BEDTools v2.25.0 [30]. In addition, we performed gene ontology (GO) enrichment and pathway analyses using the DAVID Bioinformatics Resources 6.8 [31, 32] based on human and goat background gene sets. The statistical significance was set to a $q$-value $\leq 0.05$.

\section{Confirmation of copy number variant regions by quantitative real-time PCR}

In order to evaluate the rate of false positives in our experiment, we conducted quantitative real-time PCR (qPCR) experiments to obtain an independent estimate of the copy number of four putative CNVR (CNVR_371_chr5, CNVR_506_chr6, CNVR_160_chr2 and CNVR_1229_chr21). Primers were designed with the Primer Express software (Applied Biosystems) to amplify specific regions of the ADAMTS20, BST1, NCKAP5 and TNFAIP2 genes (see Additional file 1: Table S1). As reference genes, we used the melanocortin 1 receptor (MC1R) and glucagon (GCG) genes (see Additional file 1: Table S1) loci [18, 33-35]. Quantitative PCR reactions contained 7.5 ng genomic DNA, 7.5 $\mu \mathrm{L} 2 \times$ SybrSelect Master mix (Applied Biosystems), 4.5 pmol of each forward and reverse primer, and ultrapure water to a maximum final volume of $15 \mu \mathrm{L}$. Each sample was analyzed in triplicate in order to obtain averaged copy number estimates. Reactions were loaded onto 384-well plates and run in a QuantStudio $12 \mathrm{~K}$ Flex Real-Time PCR System instrument (Applied Biosystems). The specificity of the PCR reactions was evaluated with a melting curve analysis procedure, and the efficiency (96.2-105.4\%) was assessed with standard curves. Thus, relative copy number was inferred with the qbase + software (Biogazelle, Ghent, Belgium) by using the $2^{-\Delta \Delta \mathrm{Ct}}$ approach [36]. Copy number values were calibrated by taking as a reference, four samples which, according to Goat SNP50 BeadChip data, had two copies of the investigated genomic loci.

\section{Results}

Detection of copy number variation in Murciano-Granadina goats

The initial calling with PennCNV and QuantiSNP yielded 4617 and 7750 autosomal CNV, respectively. By using the EnsembleCNV tool [29], we assigned these CNV into 1461 CNVR with refined boundaries, of which 486 (33.3\% of the total CNVR count) were detected simultaneously by PennCNV and QuantiSNP. The resulting CNVR included 78 copy gain, 353 copy loss and 55 copy gain/loss variants (Fig. 1, and Table 1) and (see Additional file 2: Table S2). The total length of the CNVR covered $95.69 \mathrm{Mb}(3.9 \%)$ of the goat autosomal genome $(2466.19 \mathrm{Mb})$, whereas their individual size ranged from $2.0 \mathrm{~kb}$ to $11.1 \mathrm{Mb}$, with an average of $196.9 \mathrm{~kb}$ (Fig. 2a and Table 1). Moreover, we found that $72.6 \%$ of the CNVR showed minimum allele frequencies lower than 0.01 , with an average frequency of $1.44 \%$ (Fig. 2b). In addition, 10 CNVR with frequencies higher than $10 \%$ were distributed over seven caprine chromosomes. With a frequency of 41\%, CNVR_1229_chr21 was the CNVR with the highest frequency in the whole dataset (see Additional file 2: Table S2). By using the BEDTools v2.25.0 program [30], 212 of the CNVR that we detected overlapped with 191 unique CNVR published by Liu et al. [18] (Fig. 1) and (see Additional file 2: Table S2). The CNVR that were detected in both studies are referred to as "shared CNVR", whereas those that were identified in our study only are referred to as "non-shared CNVR" (Fig. 1). Six of the ten "shared CNVR" with frequencies higher than 0.1 show positional concordance with six CNVR detected by Liu et al. [18] (see Additional file 2: Table S2).

\section{Functional annotation of the genes that are located in copy number variable regions}

Within the CNVR defined in our study, we detected 779 protein-coding genes according to the goat reference genome annotation (ARS1) [23] from the NCBI database (see Additional file 2: Table S2 and Additional file 3: 


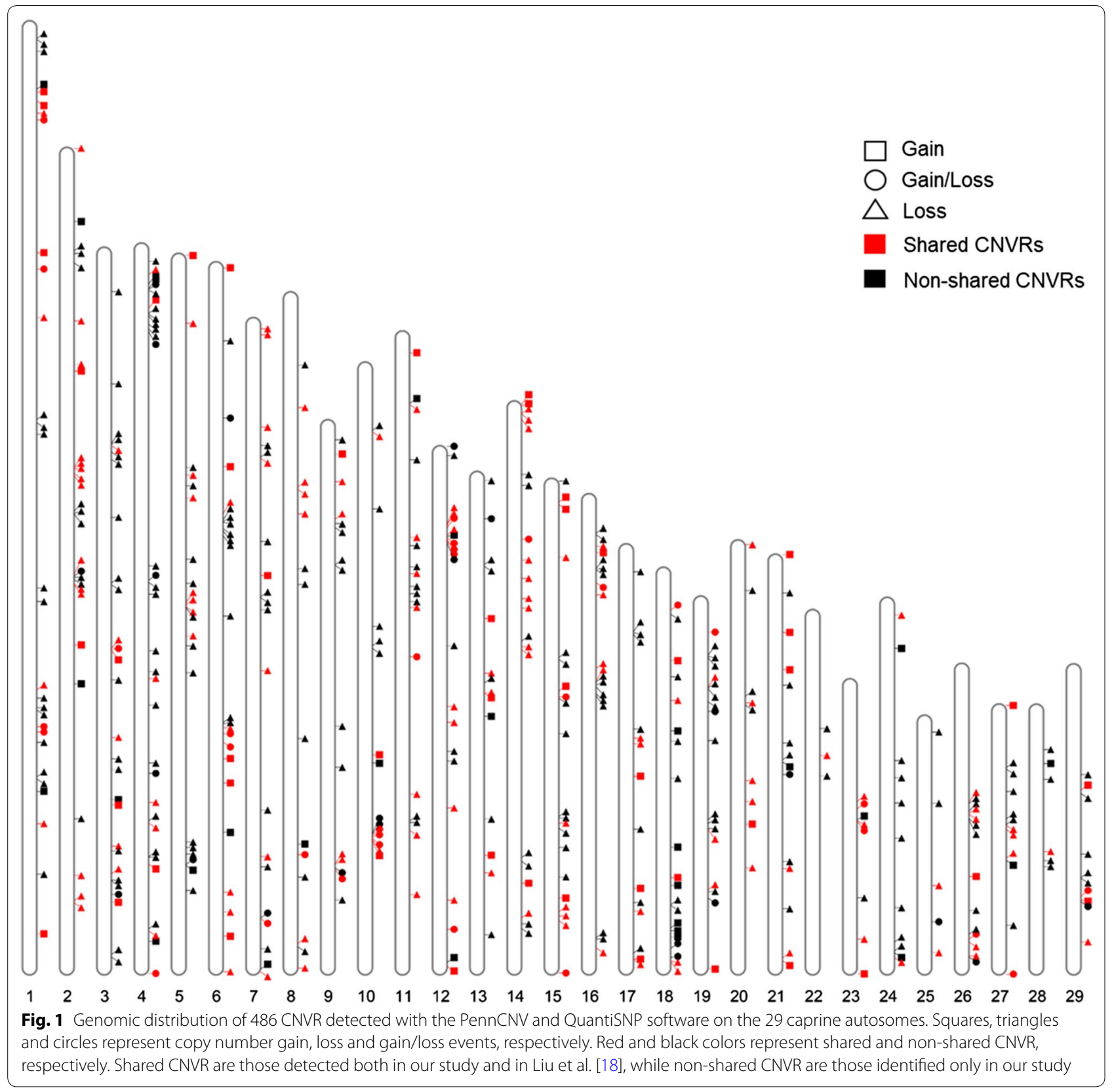

Table S3). In a survey of the diversity of CNV in goats with a worldwide distribution, Liu et al. [18] detected 1437 copy number variable genes, of which 116 were also identified in our study and are referred to as "shared copy number variable genes" (see Additional file 3: Table S3). Among the "shared copy number variable genes", the ASIP and ADAMTS20 genes are particularly relevant: they are involved in pigmentation $[6,8,17,35,37-39]$ and co-localize with selection signals detected in a worldwide sample of goats [40]. In addition, we found that about $11.4 \%$ (89) of the annotated genes that co-localize with CNVR are olfactory receptors or olfactory receptorlike genes (see Additional file 3: Table S3). Consistently, the most significantly enriched pathway was "Olfactory transduction" ( $q$-value $=1.61 \times 10^{-10}$, Table 2$)$, followed by "ABC transporter" ( $q$-value $=4.27 \times 10^{-4}$, Table 2$)$. A significant pathway related with immunity (i.e. Fc epsilon RI signaling, $q$-value $=0.02$ ) was also identified based on a human background gene set (Table 2). Several overrepresented GO terms were related with embryonic skeletal system morphogenesis $\left(q\right.$-value $\left.=1.22 \times 10^{-3}\right)$ and G-protein coupled purinergic nucleotide receptor activity 
Table 1 Main features of copy number variation regions (CNVR) detected in 1036 Murciano-Granadina goats

\begin{tabular}{lcccc}
\hline Summary statistics & Total & Gain & Loss & Gain/loss \\
\hline Total length $(\mathrm{Mb})$ & 95.69 & 26.52 & 61.17 & 8 \\
Total number of CNVR & 486 & 78 & 353 & 55 \\
Number of CNVR (<10 kb) & 1 & 1 & 0 & 0 \\
Number of CNVR (10-50 kb) & 4 & 2 & 1 & 1 \\
Number of CNVR (50-100 kb) & 152 & 25 & 113 & 14 \\
Number of CNVR (100-500 kb) & 313 & 47 & 227 & 39 \\
Number of CNVR (500 kb-1 Mb) & 10 & 0 & 9 & 1 \\
Number of CNVR ( $\geq 1 \mathrm{Mb})$ & 6 & 3 & 3 & 0 \\
Average number of SNPs per & 5.59 & 9.01 & 5.03 & 4.35 \\
$\quad$ CNVR & & & & \\
Minimum size of CNVR (kb) & 2.04 & 2.04 & 23.2 & 43.1 \\
Maximum size of CNVR (kb) & 11,124 & 11,124 & 1629.39 & 534.16 \\
Average CNVR size (kb) & 196.89 & 339.99 & 173.28 & 145.49 \\
Standard deviation of CNVR & 539.35 & 1299.49 & 156.89 & 91.51 \\
$\quad$ size (kb) & & & & \\
\hline
\end{tabular}

( $q$-value $=6.22 \times 10^{-3}$, Table 2$)$. Interestingly, the copy number variable genes were also enriched in pathways with metabolic significance, such as prolactin signaling and insulin signaling, as well as GO terms related with feeding behavior, but none of these pathways reached the significance threshold $(q$-value $\leq 0.05)$ after correction for multiple testing (see Additional file 4: Table S4). Several of the pathways outlined in Additional file 4: Table S4 play important roles in immunity (e.g. chemokine signaling, B cell receptor signaling and $\mathrm{T}$ cell receptor signaling), cancer (e.g. endometrial cancer, proteoglycans in cancer, thyroid cancer), as well as in oncogenic signaling (e.g. Ras and ErbB signaling) (see Additional file 4: Table S4), but most of them are not significant after correction for multiple testing.
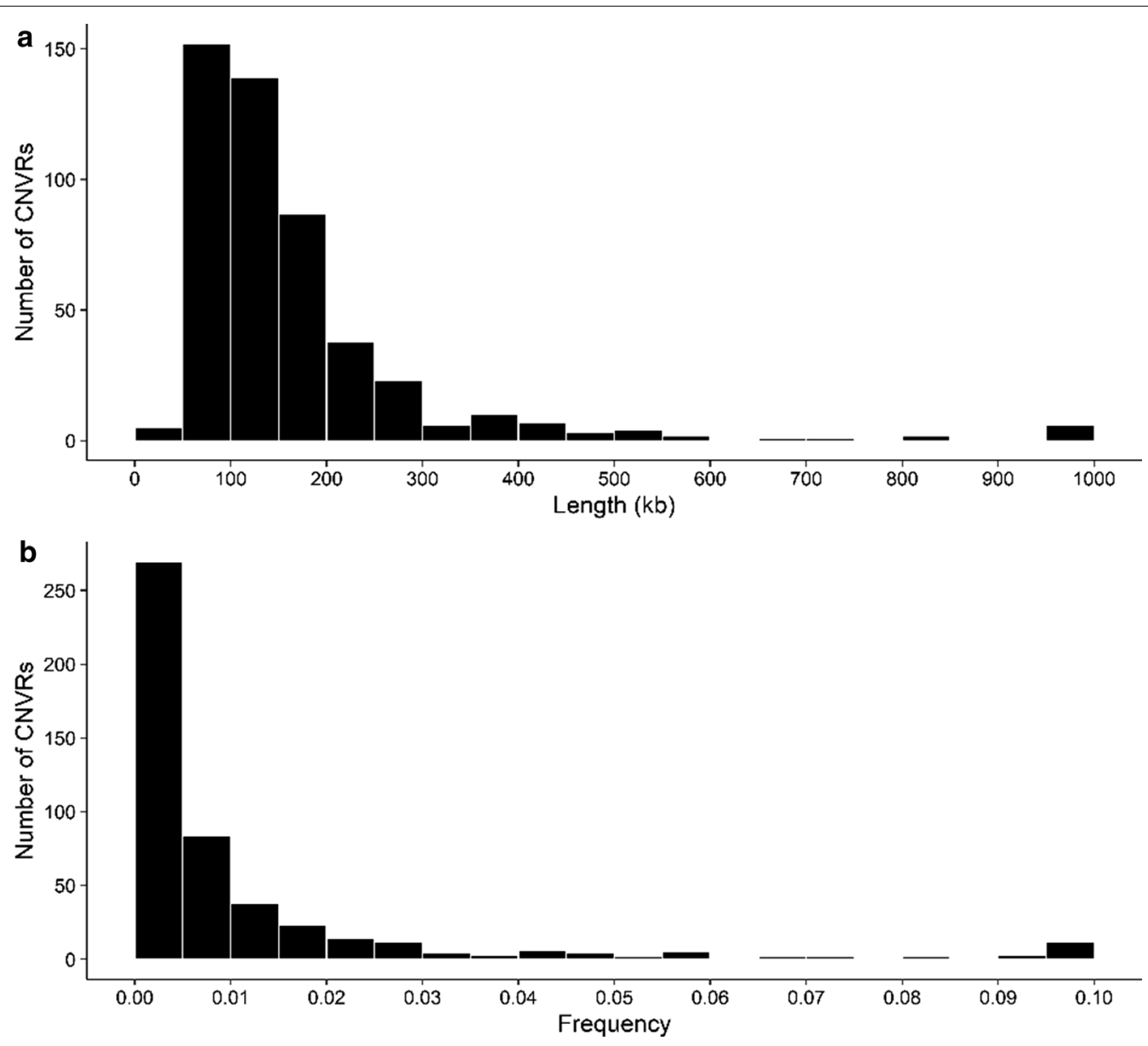

Fig. 2 Histograms displaying the distribution of CNVR according to their size (a) and frequency (b). CNVR that were longer than $1000 \mathrm{~kb}$ were included in the 1000-kb bin, whereas those with frequencies above 0.1 were grouped in the $0.1 \mathrm{bin}$. The histograms were drawn by using the ggplot2 package (http://ggplot2.tidyverse.org/) implemented in R (https://www.r-project.org/) 
Table 2 Functional enrichment of genes co-localizing with CNVR detected in 1036 Murciano-Granadina goats

\begin{tabular}{|c|c|c|c|c|c|c|c|}
\hline $\begin{array}{l}\text { Background } \\
\text { gene set }\end{array}$ & Category & ID & Term & $\begin{array}{l}\text { Number } \\
\text { of genes }\end{array}$ & Fold enrichment & $P$ value & $q$-value \\
\hline Goat & KEGG & $\operatorname{ch} \times 04740$ & Olfactory transduction & 69 & 2.33 & $1.26 \mathrm{E}-11$ & $1.61 E-10$ \\
\hline Goat & KEGG & $\operatorname{ch} \times 02010$ & $\mathrm{ABC}$ transporters & 11 & 5.27 & $3.33 \mathrm{E}-05$ & 4.27E-04 \\
\hline Goat & KEGG & chx04976 & Bile secretion & 11 & 3.90 & 4.46E-04 & 5.70E-03 \\
\hline Human & KEGG & hsa04664 & Fc epsilon RI signaling pathway & 8 & 4.71 & $1.40 \mathrm{E}-03$ & 1.76E-02 \\
\hline Human & $\mathrm{GO} / \mathrm{BP}$ & GO:0009952 & Anterior/posterior pattern specification & 12 & 5.56 & $9.36 \mathrm{E}-06$ & $1.61 \mathrm{E}-04$ \\
\hline Human & $\mathrm{GO} / \mathrm{BP}$ & GO:0048704 & Embryonic skeletal system morphogenesis & 8 & 7.60 & $7.13 \mathrm{E}-05$ & $1.22 \mathrm{E}-03$ \\
\hline Human & $\mathrm{GO} / \mathrm{BP}$ & GO:0035589 & $\begin{array}{l}\text { G-protein coupled purinergic nucleotide receptor } \\
\text { signaling pathway }\end{array}$ & 5 & 13.24 & $4.18 \mathrm{E}-04$ & $7.16 \mathrm{E}-03$ \\
\hline Human & $\mathrm{GO} / \mathrm{CC}$ & GO:0016020 & Membrane & 81 & 1.40 & $1.45 \mathrm{E}-03$ & 1.98E-02 \\
\hline Human & $\mathrm{GO} / \mathrm{MF}$ & GO:0003677 & DNA binding & 67 & 1.48 & $1.10 \mathrm{E}-03$ & 1.60E-02 \\
\hline Human & $\mathrm{GO} / \mathrm{MF}$ & GO:0045028 & $\begin{array}{l}\text { G-protein coupled purinergic nucleotide receptor } \\
\text { activity }\end{array}$ & 5 & 13.19 & $4.24 \mathrm{E}-04$ & $6.22 \mathrm{E}-03$ \\
\hline
\end{tabular}

KEGG: Kyoto Encyclopedia of Genes and Genomes pathway; GO/MF: gene ontology (GO) term related with molecular function; GO/BP: GO term related with biological process; GO/CC: GO term related with cellular component

Validation of four copy number variants by real-time quantitative polymerase chain reaction

In order to confirm our results, we selected four CNVR (i.e. CNVR_371_chr5, CNVR_506_chr6, CNVR_160_ chr2 and CNVR 1229_chr21) that co-localized with the ADAMTS20, BST1, NCKAP5 and TNFAIP2 genes, respectively (the primers used to amplify these CNVR are listed in Additional file 1: Table S1). As shown in Fig. 3, the estimated copy numbers obtained by qPCR analysis of Murciano-Granadina goat samples were: 0.93 to 2.38 copies relative to the calibrator (ADAMTS20), 1.06 to 2.96 copies (BST1), 1.51 to 2.39 copies (NCKAP5) and 1.83 to 3.28 copies (TNFAIP2). According to D'haene et al. [36], copy number estimates between 1.414 and 2.449 most likely correspond to a normal copy number of 2 , whereas any number below or above these thresholds could represent a deletion or a duplication, respectively. Thus, based on these values, evidence of copy number variation was inferred for three of the four genes analyzed by qPCR.

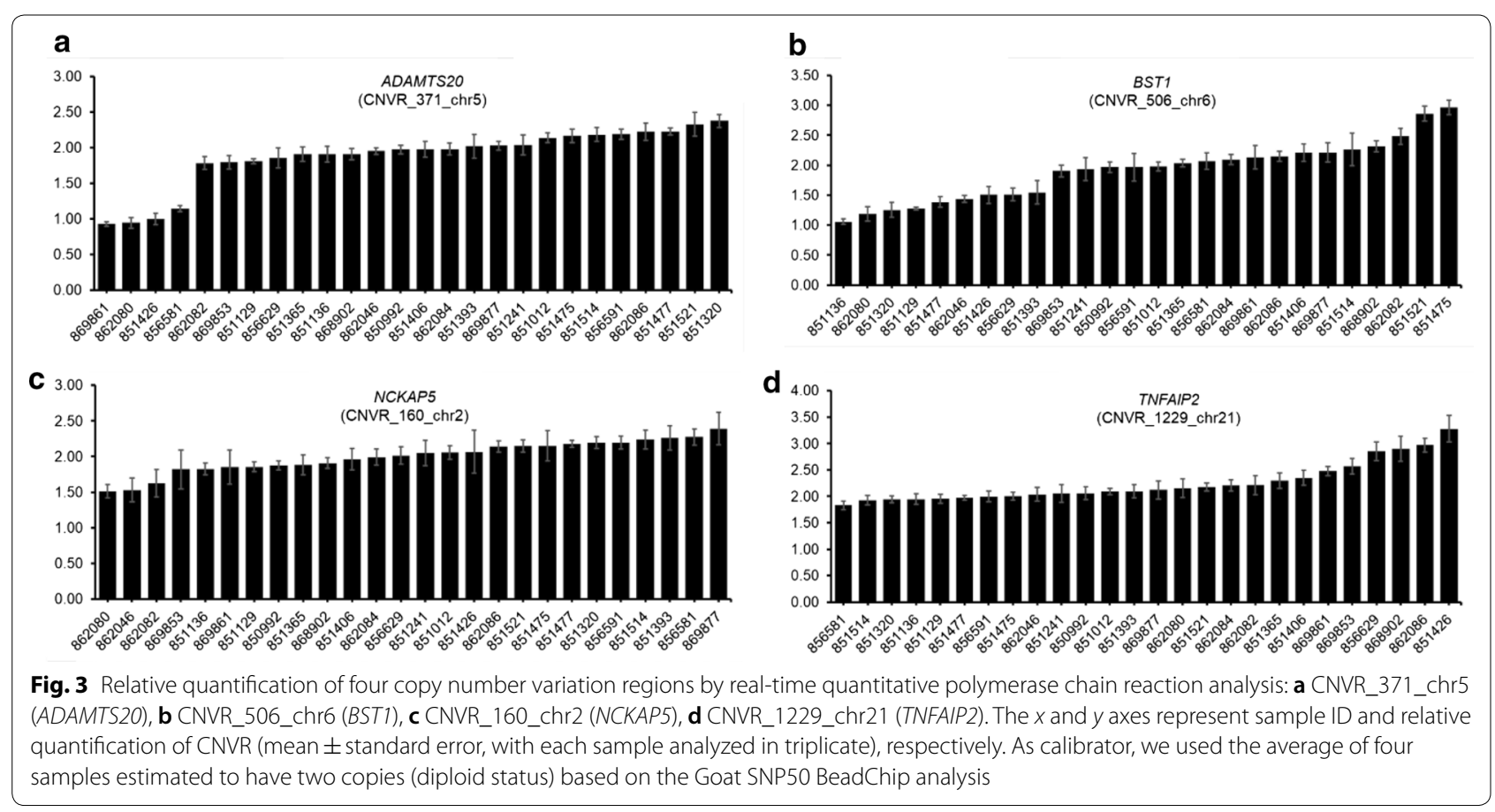




\section{Discussion}

In this work, our aim was to characterize copy number variation in Murciano-Granadina goats, a native Spanish breed used for milk production. By genotyping 1036 Murciano-Granadina goats with a SNP array, we were able to identify 486 CNVR covering $3.9 \%$ of the goat genome, whereas Liu et al. [18] identified CNVR that covered $\sim 9 \%$ of the goat genome. The latter higher percentage reported by Liu et al. [18] can be explained by the fact that they analyzed 50 breeds with different geographical origins, i.e. a composite population that is probably much more diverse than that used in our work. Besides, the pipeline that we used to identify CNVR is more stringent than that employed by Liu et al. [18], removing CNVR that were not consistently detected by PennCNV and QuantiSNP. In the literature, estimates of 4.8 to $9.5 \%$ for CNVR coverage in the human genome are reported [2]. Our results and those obtained by Liu et al. [18] are consistent with these values.

Indeed, when Liu et al. [18] calculated the CNVR length for each breed normalized by the goat genome size, their results agreed well with our estimate of $3.9 \%$. For instance, this parameter reached values of $3.94 \%$ in goats from Southeastern Africa and 3.13\% in goats from Northwestern Africa and Eastern Mediterranean, whereas it was lowest $(0.70 \%)$ for individuals from West Asia [18]. The number of CNV detected at the withinbreed level by Liu et al. [18] was on average $126 \mathrm{CNV}$ per breed and ranged from 6 to 714, whereas the average number of CNVR was only $\sim 20$ per breed [18]. Since the number of detected CNVR is proportional to population size, for most of the breeds investigated in [18], the level of within-breed CNV variation is probably underestimated. In summary, one important conclusion from our study is that the magnitude of CNV diversity at the within-breed level is likely to be much larger than that previously reported in studies that analyzed multiple populations, each represented by a small or moderate number of individuals.

Most of the CNVR that we report here ranged in size from 50 to $500 \mathrm{~kb}$, with a mean size of $196.89 \mathrm{~kb}$. Similarly, the average CNVR size reported by Liu et al. [18] was $268 \mathrm{~kb}$. Both estimates are quite large and reflect that medium-density SNP arrays are not well suited to detect small CNVR in spite of their high abundance. In cattle, the average sizes of CNVR detected with the Illumina BovineHD Genotyping BeadChip (777 K SNPs) [14], Illumina whole-genome sequencing and PacBio sequencing [41] were 66.15, 10 and $0.81 \mathrm{~kb}$, respectively. Another consistent feature of CNVR is that, in general, their frequencies are low or very low. In our study, approximately $73 \%$ of the CNVR had frequencies lower than $1 \%$ and the average frequency was $1.44 \%$. Liu et al. [18] reported lower CNVR frequencies ranging from 0.34\% (Alpine and Northern European goats) to $0.98 \%$ (Northwestern African goats). This decreased average CNVR frequency is not very significant and probably reflects differences in sampling size and the use of composite populations with multiple breeds, each one with its specific CNVR frequencies.

The CNVR detected in our study covered 779 proteincoding genes. Pathway analyses reflected a substantial enrichment of genes that are involved in olfactory perception, which is consistent with previous reports in cattle $[13,14]$. In this regard, there is an important difference between our results and those by Liu et al. [18]. Whereas in the study of Liu et al. [18], the term "sensory perception" was underrepresented among the CNV genes (fold enrichment $=0.21$ ), in our work the terms "olfactory transduction" (fold enrichment $=2.33$ ) and "G-protein coupled purinergic nucleotide receptor activity" (fold enrichment $=13.19$ ) were overrepresented, and many $\mathrm{CNV}$ genes were olfactory receptors. The two terms mentioned before are closely related because a broad array of purinergic receptors are differentially expressed in the olfactory receptor neurons that modulate odor responsiveness [42]. Moreover, purinergic nucleotides are important neuromodulators of peripheral auditory and visual sensory systems [42]. In cattle, Keel et al. [13] reported that "sensory perception of smell" and "G-protein coupled receptor signaling pathway" were significantly overrepresented in the protein-coding genes that overlapped with CNVR. Similarly, Upadhyay et al. [14] showed that "sensory perceptions of smell" and "chemical stimuli" are enriched in their set of CNV genes. A potential explanation for the underrepresentation of the "sensory perception" functional category among the genes overlapping CNV reported by Liu et al. [18] could be that in goats these genes are not well annotated yet, so the majority of them are identified with a LOC prefix and a number and, as a consequence of this, they are not correctly detected by PANTHER [43], thus biasing the results obtained in the gene ontology enrichment analysis.

Loci belonging to large multigene families might be more prone to co-localize with $\mathrm{CNV}$ because paralogous genes can act as templates in non-allelic homologous recombination events, which promote increases or reductions in copy number [44]. It should be noted that olfactory receptor genes constitute the largest gene superfamily, and in humans more than 900 genes and pseudogenes have been identified [45]. In cattle, 1071 olfactory receptor genes and pseudogenes are distributed in 49 clusters across 26 bovine chromosomes [46], and similar numbers have been reported for pigs [47]. Moreover, purifying selection against $\mathrm{CNV}$ is probably less 
intense in regions that contain olfactory-receptor genes than in genomic regions that contain genes with essential functions [48]. Interestingly, copy number changes in the olfactory receptor genes of wild and domestic mammals might have consequences on food foraging as well as on mate and predator recognition $[49,50]$.

In the set of genes that co-localize with CNVR, we also detected an enrichment of loci related with the multigene family of ATP binding cassette (ABC) transporters, a result that agrees well with previous findings in humans [51-54] and cattle [14, 56]. In mammals, ABC transporters fulfill the mission of carrying a broad array of endogenous substrates, such as amino acids, peptides, sugars, anions and hydrophobic compounds and metabolites across lipid membranes. At least $49 \mathrm{ABC}$ genes that belong to eight subfamilies have been identified in the human genome [52]. Copy number variation in the human $A B C C 4$ and $A B C C 6$ genes is associated with susceptibility to esophageal squamous cell carcinoma [51] and to the rare autosomal recessive disease pseudoxanthoma elasticum [54], respectively. Moreover, large-scale deletions of the human $A B C A 1$ gene are a causative factor for hypoalphalipoproteinemia [53], a disease that is characterized by the complete absence of the apolipoprotein AI and extremely low levels of plasma high-density lipoprotein (HDL) cholesterol. We also found a highly significant enrichment of pathways related with embryo development (anterior/posterior pattern specification, embryonic skeletal system morphogenesis), as previously reported [18]. These pathways are featured by genes that belong to the Hox multigene family of transcription factors, possibly reflecting the genomic instability of certain homeobox gene clusters as evidenced by the existence of many synteny/paralogy breakpoints and assembly gaps as outlined in comparative studies [55].

Although not significant after correction for multiple testing, we detected an enrichment of pathways with metabolic significance, such as prolactin and insulin signaling, which could have an impact on milk production and growth [57-59]. Interestingly, the comparison of our work with that of Liu et al. [18] revealed 116 protein-coding genes that co-localize with the set of shared CNVR. One of the most relevant shared genes encodes ASIP, a protein that increases the ratio of pheomelanin to eumelanin by binding to the melanocortin 1 receptor and delivering an antagonist signal that blocks the downstream expression of eumelanogenic enzymes [60]. Mutations in the ASIP gene play critical roles in animal pigmentation [39]. For instance, the causal factor of the white color typical of many sheep breeds is the ubiquitous expression of a duplicated copy of the ASIP coding sequence, which is regulated by a duplicated promoter corresponding to the itchy E3 ubiquitin protein ligase gene [6,39]. Although some studies proposed that the ASIP CNV might be associated with different pigmentation patterns in goats $[8,17,37]$, no functional assay has verified an association of $A S I P$ copy number with $A S I P$ mRNA levels. Another interesting shared copy number variable gene is $A D A M T S 20$, which was also identified in two previous CNV surveys $[17,18]$. This gene encodes a metalloproteinase with an important role in melanoblast survival by mediating Kit signaling [38] and in palatogenesis [61]. Bertolini et al. [40] performed a selection scan in white vs. colored (black and red) goats and detected a selective sweep in the ADAMTS20 gene. In the light of these results, the potential involvement of a structural variation in ADAMTS2O in goat pigmentation should be explored further. Moreover, it is worthwhile to mention that several CNVR genes have functions related with production and reproduction traits. For instance, the NCKAP5 gene, which co-localizes with CNVR_160_chr2 (frequency $=0.1$ ), is associated with milk fat percentage in cattle [62]. Taking the above evidence into account, the implication of structural chromosomal variations in the genetic determinism of traits of economic interest with a complex inheritance deserves further exploration by designing tools that allow inferring CNVR genotypes with high confidence.

\section{Conclusions}

With the PennCNV and QuantiSNP software, we detected 486 CNVR in the genome of the MurcianoGranadina breed. In a previous study [18] that used a less stringent pipeline (only PennCNV was used) and included multiple populations with small to moderate sample sizes, the average number of CNVR events per breed was $\sim 20$. One conclusion of our study is that CNV surveys, which are based on a broad array of breeds represented by only a few individuals, underestimate the true levels of the $\mathrm{CNV}$ diversity at the within-breed level. The main reason for this outcome is that since the majority of $\mathrm{CNV}$ have very low frequencies, they cannot be detected efficiently when sample size is small and, in consequence, much of the existing variation is missed. We have also found that genes that overlap with $\mathrm{CNV}$ are functionally related with olfactory transduction, embryo development, $\mathrm{ABC}$ transporters and G-protein coupled purinergic nucleotide receptor activity. Most of these genes belong to large multigene families encompassing tens, hundreds or thousands of paralogous genes that could act as substrates in non-allelic homologous recombination events, which is one of the main mechanisms generating duplications and deletions in humans and other species. Finally, we detected CNV that co-localize with the ASIP and ADAMTS20 pigmentation genes, 
which according to previous studies have been subjected to positive selection for coat color in goats.

\section{Supplementary information}

Supplementary information accompanies this paper at https://doi. org/10.1186/s12711-020-00564-4.

Additional file 1: Table S1. List of primers used in the real-time quantitative PCR experiment to validate four putative copy number variable genes.

Additional file 2: Table S2. List of copy number variation regions (CNVR) consistently detected with PennCNV and QuantiSNP in 1036 MurcianoGranadina goats.

Additional file 3: Table S3. List of copy number variable genes detected in the current work and their concordance with those reported by Liu et al. [18].

Additional file 4: Table S4. Functional enrichment of genes co-localizing with copy number variation regions detected in 1036 Murciano-Granadina goats.

\section{Acknowledgements}

The authors are indebted to the Asociación Nacional de Criadores de Caprino de Raza Murciano-Granadina (CAPRIGRAN), and especially to Miguel García García and Teresa Novo Díaz, who collected all blood samples. We are also indebted to Dr. George Liu (USDA) for providing technical advice in CNV calling.

\section{Authors' contributions}

MA, JJ, VL and JVD conceived and designed the experiment; JF-A and XS contributed to the collection of materials and reagents, $V L$ and $A M$ performed DNA extractions, AC and BC carried out DNA quality assessment and genotyping tasks, DG performed the bioinformatic analyses of the data, MGL contributed to the bioinformatic analyses of the data, AC and DG did the quantitative PCR analyses, MA and DG wrote the manuscript. All authors read and approved the final manuscript.

\section{Funding}

This research was funded by the European Regional Development Fund (FEDER)/Ministerio de Ciencia, Innovación y Universidades-Agencia Estatal de Investigación/Project Reference Grant: AGL2016-76108-R. We also acknowledge the support of the Spanish Ministry of Economy and Competitivity for the Center of Excellence Severo Ochoa 2016-2019 (SEV-2015-0533) grant awarded to the Centre for Research in Agricultural Genomics (CRAG, Bellaterra, Spain) as well as to the CERCA Programme/Generalitat de Catalunya. Dailu Guan was funded by a Ph.D. fellowship from the China Scholarship Council (CSC). María Gracia Luigi-Sierra was funded with a FPI Ph.D. grant from the Spanish Ministry of Economy and Competitivity (BES-2017-079709).

\section{Availability of data and materials}

The dataset supporting the conclusions of this article is accessible at Figshare (https://doi.org/10.6084/m9.figshare.12674357).

\section{Ethics approval and consent to participate}

The collection of blood is a routine procedure carried out by trained veterinarians working for the CAPRIGRAN association, so it does not require a permission from the Committee on Ethics in Animal and Human Experimentation of the Universitat Autònoma de Barcelona.

\section{Consent for publication}

Not applicable.

\section{Competing interests}

The authors declare that they have no competing interests.

\section{Author details}

${ }^{1}$ Centre for Research in Agricultural Genomics (CRAG), CSIC-IRTA-UAB-UB, Universitat Autònoma de Barcelona, 08193 Bellaterra, Spain. ${ }^{2}$ Departamento de
Genética, Universidad de Córdoba, 14071 Córdoba, Spain. ${ }^{3}$ Departament de Ciència Animal i dels Aliments, Facultat de Veterinària, Universitat Autònoma de Barcelona, 08193 Bellaterra, Spain. ${ }^{4}$ Department of Veterinary Medicine, University of Bari "Aldo Moro", SP. 62 per Casamassima km. 3, 70010 Valenzano, BA, Italy. ${ }^{5}$ Asociación Nacional de Criadores de Caprino de Raza Murciano-Granadina (CAPRIGRAN), 18340 Granada, Spain. ${ }^{6}$ Group of Research in Ruminants (G2R), Department of Animal and Food Science, Universitat Autònoma de Barcelona (UAB), Bellaterra, Barcelona, Spain.

Received: 2 March 2020 Accepted: 28 July 2020

Published online: 08 August 2020

\section{References}

1. Clop A, Vidal O, Amills M. Copy number variation in the genomes of domestic animals. Anim Genet. 2012;43:503-17.

2. Zarrei M, MacDonald JR, Merico D, Scherer SW. A copy number variation map of the human genome. Nat Rev Genet. 2015;16:172-83.

3. Bickhart DM, Liu GE. The challenges and importance of structural variation detection in livestock. Front Genet. 2014;5:37.

4. Wright D, Boije H, Meadows JRS, Bed'hom B, Gourichon D, Vieaud A, et al. Copy number variation in intron 1 of SOX5 causes the pea-comb phenotype in chickens. PLoS Genet. 2009:5:e1000512.

5. Chen C, Liu C, Xiong X, Fang S, Yang H, Zhang Z, et al. Copy number variation in the MSRB3 gene enlarges porcine ear size through a mechanism involving miR-584-5p. Genet Sel Evol. 2018;50:72.

6. Norris BJ, Whan VA. A gene duplication affecting expression of the ovine ASIP gene is responsible for white and black sheep. Genome Res. 2008;18:1282-93.

7. Menzi F, Keller I, Reber I, Beck J, Brenig B, Schütz E, et al. Genomic amplification of the caprine EDNRA locus might lead to a dose dependent loss of pigmentation. Sci Rep. 2016;6:28438.

8. Henkel J, Saif R, Jagannathan V, Schmocker C, Zeindler F, Bangerter E, et al. Selection signatures in goats reveal copy number variants underlying breed-defining coat color phenotypes. PLoS Genet. 2019;15:e1008536.

9. Giuffra E, Törnsten A, Marklund S, Bongcam-Rudloff E, Chardon P, Kijas $\mathrm{JMH}$, et al. A large duplication associated with dominant white color in pigs originated by homologous recombination between LINE elements flanking KIT. Mamm Genome. 2002;13:569-77.

10. Pailhoux E, Vigier B, Chaffaux S, Servel N, Taourit S, Furet JP, et al. A 11.7$\mathrm{kb}$ deletion triggers intersexuality and polledness in goats. Nat Genet. 2001;29:453-8.

11. Sundström E, Imsland F, Mikko S, Wade C, Sigurdsson S, Pielberg GR, et al. Copy number expansion of the STX17 duplication in melanoma tissue from Grey horses. BMC Genomics. 2012;13:365.

12. Letaief R, Rebours E, Grohs C, Meersseman C, Fritz S, Trouilh L, et al. Identification of copy number variation in French dairy and beef breeds using next-generation sequencing. Genet Sel Evol. 2017;49:77.

13. Keel BN, Lindholm-Perry AK, Snelling WM. Evolutionary and functional features of copy number variation in the cattle genome. Front Genet. 2016;7:207.

14. Upadhyay M, da Silva VH, Megens HJ, Visker MHPW, Ajmone-Marsan P, Bâlteanu VA, et al. Distribution and functionality of copy number variation across European cattle populations. Front Genet. 2017;8:108.

15. Hou Y, Bickhart DM, Hvinden ML, Li C, Song J, Boichard DA, et al. Fine mapping of copy number variations on two cattle genome assemblies using high density SNP array. BMC Genomics. 2012;13:376.

16. Fontanesi L, Martelli PL, Beretti F, Riggio V, Dall'Olio S, Colombo M, et al. An initial comparative map of copy number variations in the goat (Capra hircus) genome. BMC Genomics. 2010;11:639.

17. Dong Y, Zhang X, Xie M, Arefnezhad B, Wang Z, Wang W, et al. Reference genome of wild goat (capra aegagrus) and sequencing of goat breeds provide insight into genic basis of goat domestication. BMC Genomics. 2015;16:431.

18. Liu M, Zhou Y, Rosen BD, Van Tassell CP, Stella A, Tosser-Klopp G, et al. Diversity of copy number variation in the worldwide goat population. Heredity. 2018;122:636-46.

19. Nandolo W, Lamuno D, Banda L, Gondwe T, Mulindwa H, Nakimbugwe H, et al. Distribution of copy number variants in the genomes of East African 
goat breeds. In: Proceedings of the 11 th world congress on genetics applied to livestock production, 11-16 Feb 2018, Auckland. 2018.

20. Miller SA, Dykes DD, Polesky HF. A simple salting out procedure for extracting DNA from human nucleated cells. Nucleic Acids Res. 1988;16:1215.

21. Tosser-Klopp G, Bardou P, Bouchez O, Cabau C, Crooijmans R, Dong Y, et al. Design and characterization of a $52 \mathrm{~K}$ SNP Chip for goats. PLoS One. 2014;9:e86227.

22. Attiyeh EF, Diskin SJ, Attiyeh MA, Mossé YP, Hou C, Jackson EM, et al. Genomic copy number determination in cancer cells from single nucleotide polymorphism microarrays based on quantitative genotyping corrected for aneuploidy. Genome Res. 2009;19:276-83.

23. Bickhart DM, Rosen BD, Koren S, Sayre BL, Hastie AR, Chan S, et al. Singlemolecule sequencing and chromatin conformation capture enable de novo reference assembly of the domestic goat genome. Nat Genet. 2017:49:643-50.

24. Wang K, Li M, Hadley D, Liu R, Glessner J, Grant SF, et al. PennCNV: an integrated hidden Markov model designed for high-resolution copy number variation detection in whole-genome SNP genotyping data. Genome Res. 2007:17:1665-74.

25. Colella S, Yau C, Taylor JM, Mirza G, Butler H, Clouston P, et al. QuantiSNP: an Objective Bayes Hidden-Markov Model to detect and accurately map copy number variation using SNP genotyping data. Nucleic Acids Res. 2007;35:2013-25.

26. Winchester L, Yau C, Ragoussis J. Comparing CNV detection methods for SNP arrays. Brief Funct Genomics. 2009;8:353-66.

27. Pinto D, Darvishi K, Shi X, Rajan D, Rigler D, Fitzgerald T, et al. Comprehensive assessment of array-based platforms and calling algorithms for detection of copy number variants. Nat Biotechnol. 2011;29:512-20.

28. Diskin SJ, Li M, Hou C, Yang S, Glessner J, Hakonarson H, et al. Adjustment of genomic waves in signal intensities from whole-genome SNP genotyping platforms. Nucleic Acids Res. 2008;36:e126.

29. Zhang Z, Cheng H, Hong X, Di Narzo AF, Franzen O, Peng S, et al. EnsembleCNV: an ensemble machine learning algorithm to identify and genotype copy number variation using SNP array data. Nucleic Acids Res. 2019;47:e39.

30. Quinlan AR, Hall IM. BEDTools: a flexible suite of utilities for comparing genomic features. Bioinformatics. 2010;26:841-2.

31. Huang DW, Sherman BT, Lempicki RA. Systematic and integrative analysis of large gene lists using DAVID bioinformatics resources. Nat Protoc. 2009;4:44-57.

32. Huang DW, Sherman BT, Lempicki RA. Bioinformatics enrichment tools: paths toward the comprehensive functional analysis of large gene lists. Nucleic Acids Res. 2009;37:1-13.

33. Ballester M, Castelló A, Ibáñez E, Sánchez A, Folch JM. Real-time quantitative PCR-based system for determining transgene copy number in transgenic animals. Biotechniques. 2004;37:610-3.

34. Ramayo-Caldas Y, Castelló A, Pena RN, Alves E, Mercadé A, Souza CA, et al. Copy number variation in the porcine genome inferred from a $60 \mathrm{~K} \mathrm{SNP}$ BeadChip. BMC Genomics. 2010;11:593.

35. Fontanesi L, Beretti F, Riggio V, Gómez González E, Dall'Olio S, Davoli $R$, et al. Copy number variation and missense mutations of the agouti signaling protein (ASIP) gene in goat breeds with different coat colors. Cytogenet Genome Res. 2009;126:333-47.

36. D'haene B, Vandesompele J, Hellemans J. Accurate and objective copy number profiling using real-time quantitative PCR. Methods. 2010;50:262-70

37. Zhang B, Chang L, Lan X, Asif N, Guan F, Fu D, et al. Genome-wide definition of selective sweeps reveals molecular evidence of trait-driven domestication among elite goat (Capra species) breeds for the production of dairy, cashmere, and meat. GigaScience. 2018;7:giy105.

38. Silver DL, Hou L, Somerville R, Young ME, Apte SS, Pavan WJ. The secreted metalloprotease ADAMTS20 is required for melanoblast survival. PLoS Genet. 2008:4:e1000003.

39. Georges M, Charlier C, Hayes B. Harnessing genomic information for livestock improvement. Nat Rev Genet. 2019;20:135-56.

40. Bertolini F, Servin B, Talenti A, Rochat E, Kim ES, Oget C, et al. Signatures of selection and environmental adaptation across the goat genome postdomestication. Genet Sel Evol. 2018;50:57.

41. Couldrey C, Keehan M, Johnson T, Tiplady K, Winkelman A, Littlejohn MD, et al. Detection and assessment of copy number variation using PacBio long-read and Illumina sequencing in New Zealand dairy cattle. J Dairy Sci. 2017;100:5472-8

42. Hegg CC, Greenwood D, Huang W, Han P, Lucero MT. Activation of purinergic receptor subtypes modulates odor sensitivity. J Neurosci. 2003;23:8291-301.

43. Mi H, Muruganujan A, Huang X, Ebert D, Mills C, Guo X, Thomas PD. Protocol update for large-scale genome and gene function analysis with the PANTHER classification system (v.14.0). Nat Protoc. 2019;14:703-21.

44. Hastings PJ, Lupski JR, Rosenberg SM, Ira G. Mechanisms of change in gene copy number. Nat Rev Genet. 2009;10:551-64.

45. Glusman G, Yanai I, Rubin I, Lancet D. The complete human olfactory subgenome. Genome Res. 2001;11:685-702.

46. Lee K, Nguyen DT, Choi M, Cha SY, Kim JH, Dadi H, et al. Analysis of cattle olfactory subgenome: the first detail study on the characteristics of the complete olfactory receptor repertoire of a ruminant. BMC Genomics. 2013;14:596

47. Nguyen DT, Lee K, Choi H, Choi MK, Le MT, Song N, et al. The complete swine olfactory subgenome: expansion of the olfactory gene repertoire in the pig genome. BMC Genomics. 2012;13:584.

48. Young JM, Endicott RM, Parghi SS, Walker M, Kidd JM, Trask BJ. Extensive copy-number variation of the human olfactory receptor gene family. Am J Hum Genet. 2008;83:228.

49. Paudel Y, Madsen O, Megens HJ, Frantz LAF, Bosse M, Crooijmans RPMA, et al. Copy number variation in the speciation of pigs: a possible prominent role for olfactory receptors. BMC Genomics. 2015;16:330.

50. Rinker DC, Specian NK, Zhao S, Gibbons JG. Polar bear evolution is marked by rapid changes in gene copy number in response to dietary shift. Proc Natl Acad Sci USA. 2019;116:13446-51.

51. Sun Y, Shi N, Lu H, Zhang J, Ma Y, Qiao Y, et al. ABCC4 copy number variation is associated with susceptibility to esophageal squamous cell carcinoma. Carcinogenesis. 2014:35:1941-50.

52. Vasiliou V, Vasiliou K, Nebert DW. Human ATP-binding cassette (ABC) transporter family. Hum Genomics. 2009;3:281-90.

53. Dron JS, Wang J, Berberich AJ, lacocca MA, Cao H, Yang P, et al. Largescale deletions of the $A B C A 1$ gene in patients with hypoalphalipoproteinemia. J Lipid Res. 2018;59:1529-35.

54. Kringen MK, Stormo C, Berg JP, Terry SF, Vocke CM, Rizvi S, et al. Copy number variation in the ATP-binding cassette transporter ABCC6 gene and $A B C C 6$ pseudogenes in patients with pseudoxanthoma elasticum. Mol Genet Genomic Med. 2015;3:233-7.

55. Wilming LG, Boychenko V, Harrow JL. Comprehensive comparative homeobox gene annotation in human and mouse. Database. 2015;2015:bav091.

56. Liu GE, Hou Y, Zhu B, Cardone MF, Jiang L, Cellamare A, et al. Analysis of copy number variations among diverse cattle breeds. Genome Res. 2010;20:693-703.

57. Fujita S, Rasmussen BB, Cadenas JG, Grady JJ, Volpi E. Effect of insulin on human skeletal muscle protein synthesis is modulated by insulin-induced changes in muscle blood flow and amino acid availability. Am J Physiol Endocrinol Metab. 2006;291:E745-54.

58. Bequette BJ, Kyle CE, Crompton LA, Buchan V, Hanigan MD. Insulin regulates milk production and mammary gland and hind-leg amino acid fluxes and blood flow in lactating goats. J Dairy Sci. 2001:84:241-55.

59. Freeman ME, Kanyicska B, Lerant A, Nagy G. Prolactin: structure, function, and regulation of secretion. Physiol Rev. 2000;80:1523-631.

60. Nasti TH, Timares L. MC1R, eumelanin and pheomelanin: their role in determining the susceptibility to skin cancer. Photochem Photobiol. 2015;91:188-200.

61. Wolf ZT, Brand HA, Shaffer JR, Leslie EJ, Arzi B, Willet CE, et al. Genomewide association studies in dogs and humans identify ADAMTS20 as a risk variant for cleft lip and palate. PLoS Genet. 2015;11:e1005059.

62. Jiang J, Ma L, Prakapenka D, VanRaden PM, Cole JB, Da Y. A large-scale genome-wide association study in U.S. Holstein cattle. Front Genet. 2019;10:420.

\section{Publisher's Note}

Springer Nature remains neutral with regard to jurisdictional claims in published maps and institutional affiliations. 